\title{
Detection of atrial shunt lesions with a single echocardio- graphic parameter
}

\author{
Varius Dannenberg · Georg Goliasch · Christian Hengstenberg · Thomas Binder · Harald Gabriel · \\ Matthias Schneider
}

Received: 14 December 2019 / Accepted: 7 April 2020 / Published online: 30 April 2020

(C) The Author(s) 2020

\begin{abstract}
Summary
Background Unrepaired left to right atrial shunt lesions can cause significant right ventricular (RV) volume overload. The parameter pulmonary to systemic shunt volume ratio (Qp:Qs) has been shown to detect even small differences between left and right ventricular stroke volume; however, four parameters are needed for its calculation. This study was carried out to evaluate the accuracy of the single parameter right ventricular outflow tract (RVOT) velocity time integral (VTI) to identify atrial shunt lesions.

Methods All patients who underwent transesophageal echocardiography (TEE) examination at this institution between 1 January 2013 and 1 January 2018 were retrospectively analyzed. The RVOT-VTI was measured in the transthoracic echocardiography performed immediately before each TEE. The diagnostic accuracy for detection of atrial shunt lesions was tested.

Results A total of 2797 patients with a median age of 67 years (interquartile range, IQR 54-77 years) were included in the final analysis. A total of 113 (4\%) patients had a relevant atrial shunt lesion. The mean RVOTVTI of the shunt group was $25 \mathrm{~cm}(S D \pm 8.1 \mathrm{~cm})$ and was significantly higher than that of the non-shunt group with $17 \mathrm{~cm}(\mathrm{SD} \pm 4.8 \mathrm{~cm})(p<0.001)$. The area under the curve (AUC) was 0.81. A total of 106 pa-
\end{abstract}

Electronic supplementary material The online version of this article (https://doi.org/10.1007/s00508-020-01659-0) contains supplementary material, which is available to authorized users.

V. Dannenberg · G. Goliasch, MD, PhD .

C. Hengstenberg, MD · T. Binder, MD · H. Gabriel, MD ·

M. Schneider, MD $(\bowtie)$

Department of Internal Medicine II, Medical University of Vienna, Waehringer Guertel 18-20, 1090 Vienna, Austria matthias.schneider@meduniwien.ac.at tients $(93.8 \%)$ of the shunt group had a VTI of $\geq 16 \mathrm{~cm}$. If the RVOT-VTI was $<16 \mathrm{~cm}$, the negative predictive value was $99.3 \%$. If the RVOT-VTI was $\geq 25 \mathrm{~cm}, 22 \%$ of patients proved to have a significant shunt lesion.

Conclusion In this large retrospective analysis it could be shown that a low RVOT-VTI predicted the absence of significant atrial shunt lesions, while a high RVOTVTI predicted the presence. The parameter should be applied in all patients with suspected atrial shunt lesions where calculation of Qp:Qs is impossible.

Keywords RVOT - Right ventricular outflow tract · VTI · Velocity time integral · Atrial septal defect

\section{Introduction}

Unrepaired atrial left to right shunt lesions can lead to significant right heart volume overload. Symptoms such as palpitations, chest pain and shortness of breath are caused by right heart failure and usually occur at a relatively late stage with already dilated right ventricular dimensions and often pulmonary hypertension [1-3]. Therefore, it is crucial to discover early signs of left to right shunts before symptoms develop.

The most common atrial left-to-right shunts include the following defects: ostium secundum atrial septal defect (ASD II), sinus venosus defect (SVD), ostium primum atrial septal defect (ASD I), coronary sinus defect and anomalous pulmonary venous connection [4]. Atrial shunt lesions can occur in combination with a patent foramen ovale (PFO) [5].

Some patients present with electrocardiogram (ECG) changes, such as (in)complete right bundle branch block or they have a fixed split-second heart sound; however, these findings are unspecific [6]. Transthoracic echocardiography (TTE) is the firstline imaging modality applied when there is suspi- 
cion of a shunt lesion [7]. Due to low blood flow velocity and the thin interatrial septum, the ASD itself or the shunted blood flow can be missed in TTE [8]. Echocardiographers rely on the presence of secondary signs for right ventricular volume overload, such as right ventricular size and function, presence of tricuspid regurgitation, signs of pulmonary hypertension, and the pulmonary blood flow $[7,9,10]$. For the evaluation of a suspected shunt lesion in TTE, the ratio of the pulmonary to systemic shunt volume (Qp:Qs) is an established method [11, 12]. Due to left-to-right shunting, there is an increased blood flow through the pulmonary circulation in these patients. Kitabatake et al. could show that the Qp:Qs was $0.99 \pm 0.05$ in healthy subjects [13]. If the ratio is greater than one, a left to right shunt should be considered [14, 15]. The pitfall of this method is the need of four parameters for its calculation: LVOT (left ventricular outflow tract) diameter, RVOT (right ventricular outflow tract) diameter, and the VTI of both outflow tracts (supplementary material 1). Particularly the assessment of the diameters of the outflow tracts can be technically challenging due to image quality [16, 17]. A falsely measured outflow tract diameter leads to wrong assumptions.

We sought to determine if a low VTI can exclude a significant atrial shunt lesion and if a high VTI can be a hint for its presence. RVOT-VTI could be a new diagnostic parameter in TTE for the diagnosis of atrial shunt lesions in those patients where the outflow tract diameters cannot be measured reliably and therefore calculation of Qp:Qs is impossible.

\section{Methods}

Gold standard for the imaging of the interatrial septum and for the diagnosis of atrial shunts is transesophageal echocardiography (TEE) [7]. A retrospective analysis was performed including all patients who received a TEE examination at this institution between 1 January 2013 and 1 January 2018. In addition, a TTE examination (which included a pulsed-wave (PW) Doppler signal in the RVOT) on the same day or in close time interval before the transesophageal echocardiography (TEE) was warranted for inclusion.

The study was conducted in accordance with the amended Declaration of Helsinki. The ethics committee of the Medical University of Vienna approved the conduct of the study (EK\# 1427/2018).

The presence of any atrial shunt lesion, the type, and significance were recorded in the defined population. The RVOT VTI of patients without shunt lesions, with non-significant shunt lesions, and with significant shunt lesions were compared. The value of the RVOT VTI for the detection of a significant left to right shunt as a single parameter was evaluated. The analysis was done by postprocessing of ultrasound loops of the cardiology department at the tertiary center.
We excluded all patients with obvious other reasons for high RVOT VTI signals, such as:

- any degree of pulmonary stenosis

- at least moderate pulmonary regurgitation

- more than moderate tricuspid regurgitation

We also excluded those patients with more than mildly reduced right ventricular function (RVF) since there might be false-low RVOT VTI due to reduced cardiac output.

Pulmonary and tricuspid regurgitation were graded by visual quantification of the color Doppler jet. Semiquantitative assessment of RVF was performed by experienced readers using multiple acoustic windows for visual assessment (eyeballing) as well as tissue Doppler imaging of the basal free lateral wall of the RV (S') and/or tricuspid annular plane systolic excursion (TAPSE), as suggested by the guidelines [18, 19]. The RVF was graded as normal, mildly, moderately, and severely reduced.

\section{Classification of shunts}

A shunt ratio of $\geq 1.2$ for Qp:Qs and/or a shunt diameter of $>3 \mathrm{~mm}$ were chosen for a shunt to be considered relevant in this study. Few studies have investigated shunt ratios and their impact on the clinical course of patients. From a physiological perspective, a shunt ratio of 1:1 is normal. Based on the data of Engelfriet at al. a ratio of $\geq 1.5$ is considered a relevant shunt which should be considered for surgery/intervention $[15,20]$. In this study we did not aim at detecting volume overload in right ventricles but to investigate the feasibility of a new parameter to uncover the presence of a left to right shunt. With 1.2, we therefore chose a ratio below 1.5 and above 1 to define the presence of a shunt lesion.

Statistical methods were independent sample T-tests and receiver operating characteristics (ROC) curves with area under the curve (AUC). Feasible cutoff values and the Youden index were defined. Sensitivity, specificity as well as positive and negative predictive value were calculated. Regarding optimal VTI cut-off, to find the best diagnostic value for the upper and lower cut-off values for shunt detection and shunt exclusion, we chose the first VTI value with a sensitivity or specificity of $>90 \%$. A $p$ value $\leq 0.05$ was considered statistically significant. The SPSS Version 24 (SPSS, IBM, Armonk, NY, USA) was used for all analyses.

\section{Results}

Between 1 January 2013 and 1 January 2018 a total of 4442 TEE examinations were performed at this department. If a patient received more than one TEE, we included the first examination and deleted the other(s). All patients with more than mildly reduced right ventricular function as well as those with significant right 


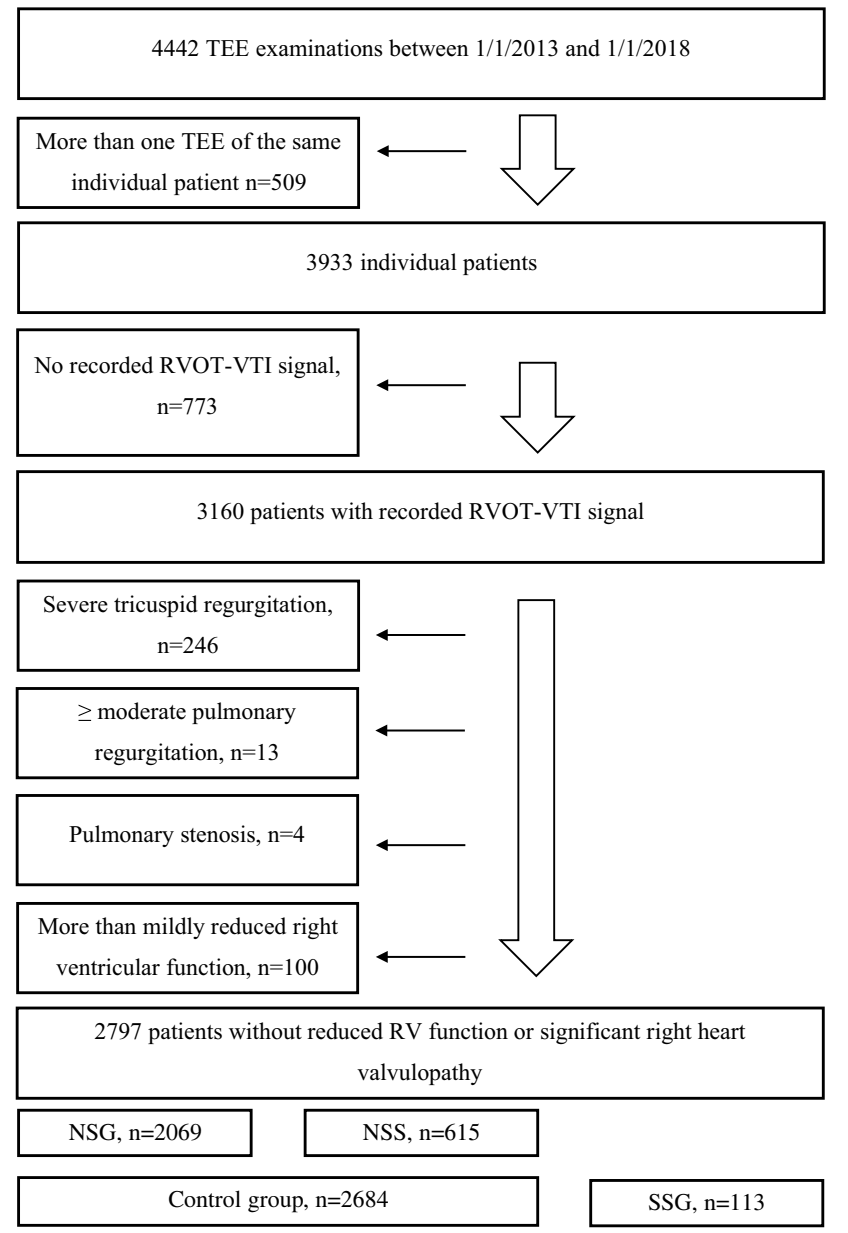

Fig. 1 STROBE (strengthening the reporting of observational studies in epidemiology) algorithm showing patient selection. TEE transesophageal echocardiography examination, $R V O T$ right ventricular outflow tract, VTI velocity time integral, $R V$ right ventricle, NSG no shunt group, NSS no significant shunt, SSG significant shunt group

heart valvulopathy were excluded to allow for unbiased evaluation of RVOT-VTI. A total of 2797 patients (median age 67 years, interquartile range 54-77 years) remained for the final analysis (Fig. 1).

Atrial shunts were found in 728 (26\%) patients. This preliminary shunt group was composed of patients with small iatrogenic ASDs after interventions with transseptal puncture $(n=27)$, PFO $(n=568)$, ASD II $(n=104)$, both PFO and ASD II $(n=14)$, ASD I $(n=2)$, and superior SVD $(n=13)$. Inferior sinus venosus ASDs or unroofed coronary sinus defects were not present. In this cohort, there were no patients with ventricular septal defects (VSD) or patent ducts (PDA).

Subsequently, all patients without significant shunt lesions were sorted into the non-significant shunt group (NSS). Shunts with Qp:Qs $\geq 1.2$ were directly added to the significant shunt group (SSG). If a Qp:Qs was not reported but the size of the ASD, ASDs $\leq 3 \mathrm{~mm}$ were added to the NSS. A total of 113 patients were assigned to the SSG. Furthermore, 2069 patients without shunts were assigned to the no-shunt group (NSG).

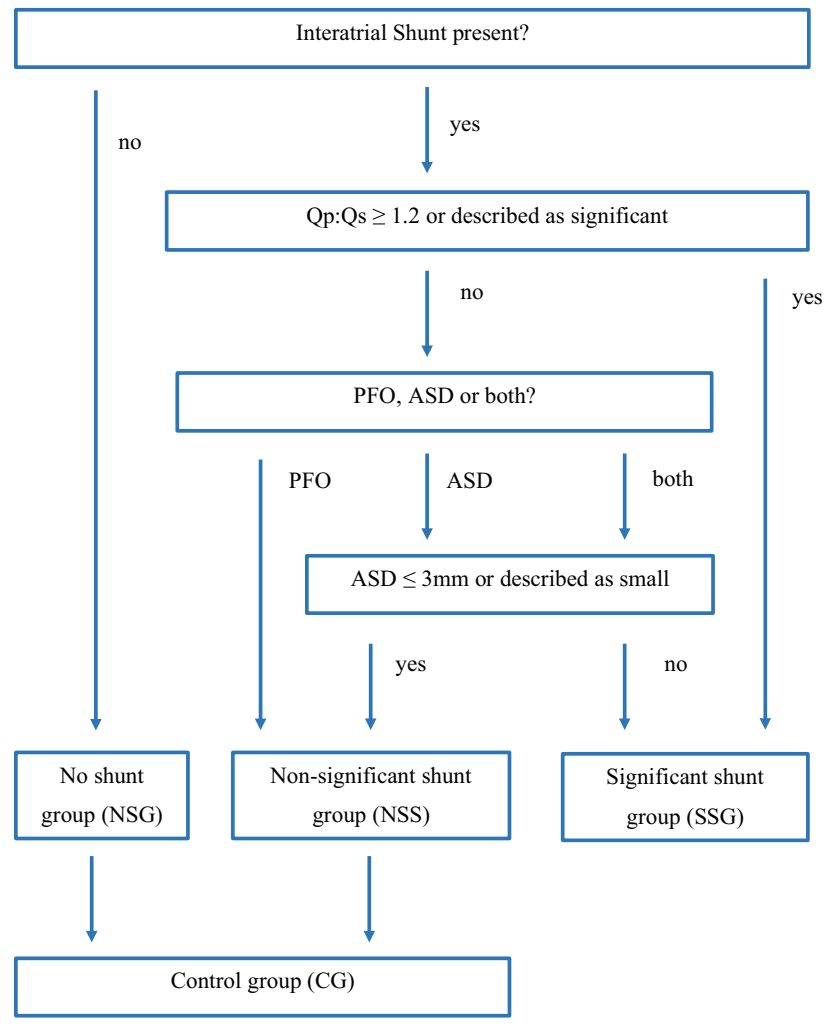

Fig. 2 Presence of an interatrial shunt: Qp:Qs indicates the ratio of pulmonary to systemic flow. Qp:Qs ratio of pulmonary to systemic flow, PFO patent foramen ovale, $A S D$ atrial septal defect, NSG no shunt group, NSS non-significant shunt group; significant shunt group, CG control group

The NSG and the NSS together were defined as the control group (CG) (Fig. 2).

Mean RVOT-VTI of the SSG with $25 \mathrm{~cm}(\mathrm{SD} \pm 8.1)$ was significantly higher than that of the CG with $17 \mathrm{~cm}$ $(\mathrm{SD} \pm 4.8)(p<0.001)$ (Table 1$)$.

The ROC curve was calculated for the VTI in all patients, the AUC was 0.819 (95\% CI 0.776-0.862). The Youden Index indicated a RVOT-VTI of $21 \mathrm{~cm}$ as the value with best sensitivity (72.6\%) and specificity (76.8\%) for the diagnosis of a significant shunt lesion.

Additional calculations were performed for the extreme cut-offs $16 \mathrm{~cm}$ and $25 \mathrm{~cm}$. A total of 106 patients $(93.8 \%)$ of the SSG had a VTI of $\geq 16 \mathrm{~cm}$. If VTI was $<16 \mathrm{~cm}$, negative predictive value was $99.3 \%$. Only 246 patients $(8.8 \%)$ had a VTI of $\geq 25 \mathrm{~cm}$. Of these patients $54(22 \%)$ had a significant shunt lesion (Table 2$)$.

\section{Discussion}

In this large retrospective study analyzing 2797 patients including 113 with significant atrial shunt lesions, those patients with relevant atrial shunts had a significantly higher RVOT-VTI than those without a shunt lesion. Therefore, RVOT-VTI should be applied as a single parameter in those patients, where Qp:Qs calculation is impossible due to image quality. 
Table 1 Baseline and echo characteristics for significant shunt group and control group

\begin{tabular}{|c|c|c|c|}
\hline Variable & Significant shunt group & Control group & $p$-value \\
\hline Age, years $( \pm S D)$ & $49.7( \pm 18.7)$ & $64.7( \pm 16.0)$ & $<0.001$ \\
\hline Female sex, $n(\%)$ & $51(45)$ & $1128(42)$ & 0.519 \\
\hline VTI-RVOT, mean ( $\pm S D)$ & $25.04( \pm 8.1)$ & $17.14( \pm 4.8)$ & $<0.001$ \\
\hline \multicolumn{4}{|l|}{ Left ventricular function } \\
\hline$<$ moderately reduced LVF, $n(\%)$ & $56(100)$ & 1427 (94.3) & 0.067 \\
\hline$\geq$ moderately reduced LVF, $n(\%)$ & $0(0)$ & $86(5.7)$ & 0.067 \\
\hline \multicolumn{4}{|l|}{ Right ventricular function } \\
\hline Normal RVF, $n(\%)$ & $62(98.4)$ & $1589(97.7)$ & 0.718 \\
\hline Mildly reduced RVF, $n(\%)$ & $1(1.6)$ & $37(2.3)$ & 0.718 \\
\hline \multicolumn{4}{|l|}{ Right ventricular size } \\
\hline Normal sized RV, $n(\%)$ & $26(40.0)$ & $1685(97.9)$ & $<0.001$ \\
\hline Mildly enlarged RV, $n(\%)$ & $18(27.7)$ & $30(1.7)$ & $<0.001$ \\
\hline$\geq$ moderately enlarged RV, $n(\%)$ & $21(32.3)$ & $7(0.4)$ & $<0.001$ \\
\hline \multicolumn{4}{|l|}{ Tricuspid valve regurgitation } \\
\hline No TR, $(\%)$ & $0(0)$ & $21(1.0)$ & 0.368 \\
\hline Mild TR, $n(\%)$ & $45(71.4)$ & $1302(79.0)$ & 0.152 \\
\hline Mild to moderate TR, $n(\%)$ & $12(19.0)$ & $211(13.0)$ & 0.148 \\
\hline Moderate TR, $n(\%)$ & $6(9.5)$ & $115(7)$ & 0.438 \\
\hline
\end{tabular}

Table 2 Diagnostic accuracy of different cut-off values for RVOT-VTI $\geq 16 \mathrm{~cm}, \geq 21 \mathrm{~cm}$, and $\geq 25 \mathrm{~cm}$

\begin{tabular}{|l|l|l|l|}
\hline$V T I \geq 16 \mathrm{~cm}$ & SSG & CG & Total \\
\hline Positive & 106 & 1656 & 1762 \\
\hline Negative & 7 & 1028 & 1035 \\
\hline Total & 113 & 2684 & 2797 \\
\hline$V T I \geq 21 \mathrm{~cm}$ & $S S G$ & $C G$ & Total \\
\hline Positive & 82 & 623 & 705 \\
\hline Negative & 31 & 2061 & 2092 \\
\hline Total & 113 & 2684 & 2797 \\
\hline$V T I \geq 25 \mathrm{~cm}$ & SSG & $C G$ & Total \\
\hline Positive & 54 & 192 & 246 \\
\hline Negative & 59 & 2492 & 2551 \\
\hline Total & 113 & 2684 & 2797 \\
\hline VTI velocity time integral, SSG significant shunt group, CG control group \\
\hline
\end{tabular}

Any unexplained dilatation of the right heart chambers must raise suspicion for the presence of significant atrial shunt lesions. Careful TTE imaging of the interatrial septum via 2D echocardiography from atypical views, the application of color Doppler imaging as well as intravenous contrast agents help with the diagnosis. In cases with limited image quality, the morphology of the interatrial septum can remain inconclusive in TTE. Due to large shunt areas and therefore slow blood flow through the lesion, color Doppler imaging can be inconclusive as well. In these patients, significant blood flow from left to right can inhibit right heart contrast agent from crossing to the left heart. The typical wash-out phenomenon can be very clear via TEE but can be difficult to detect in TTE. This may leave echocardiographers with the iso- lated finding of a dilated right heart and no further information on the possible presence of a shunt lesion. With Qp:Qs, a reliable echocardiographic hemodynamic parameter is available for further evaluation and for the decision making if the particular patient is referred to TEE, where a definitive diagnosis can be made. Unfortunately, especially the correct measurement of the diameter of the RVOT is impossible in numerous patients due to image quality. At the same time, RVOT-VTI can be measured in almost all patients in the parasternal long or short axis, in the apical inflow-outflow view, or from subcostal angulation without the need for good image quality. In this analysis, we sought to evaluate if the single parameter RVOT-VTI can distinguish between patients with and without atrial shunt lesions.

With a mean of $25 \mathrm{~cm}$ and $17 \mathrm{~cm}$ respectively, RVOT-VTI was significantly $(p<0.001)$ higher in patients with relevant atrial shunt lesions than in those without. In ROC analysis, AUC to differentiate between the two groups was 0.819 . The Youden index determined a VTI of $21 \mathrm{~cm}$ as the best value regarding specificity and sensitivity; however, neither specificity (76.8\%) nor sensitivity (72.6\%) are good enough to use RVOT-VTI of $21 \mathrm{~cm}$ as diagnostic guidance in clinical practice. We therefore analyzed the extreme cut-offs at the lower and the upper end of the VTI spectrum.

Considering a VTI of $16 \mathrm{~cm}$, only 7 of the 113 significant shunt lesions fell below this cut-off, negative predictive value was $99.3 \%$. It can be concluded that a significant shunt lesion is unlikely if VTI is $<16 \mathrm{~cm}$.

The RVOT-VTI values of patients with an ASD I or a superior SVD lesion were high with $31.5 \mathrm{~cm}$ and 
$31.8 \mathrm{~cm}$, respectively. In those patients with a VTI of $25 \mathrm{~cm}$ and more, $22 \%$ of patients proved to have a significant shunt lesion. In the light of these data, a high RVOT-VTI signal in combination with a dilated right heart should prompt the suspicion for the presence of a significant atrial shunt lesion, if other reasons for high RVOT-VTI signals have been previously ruled out. A TEE should be carried out as a further diagnostic test in these patients.

Apart from the validation of RVOT-VTI as a diagnostic parameter for the detection of shunt lesions, this work also allows a closer look at the prevalence of shunt lesions at this tertiary center in Austria. The analysis includes all TEE examinations between 1 January 2013 and 1 January 2018. In total, 113 significant shunt lesions were found. In this adult cohort with a median age of 67 years, ASD II was the most frequent shunt lesion with $79 \%$ of cases, followed by superior SVD in $11.5 \%$ of cases.

Finally, the age of the patients was investigated and the mean age in the significant shunt group was 49 years. This shows that atrial shunt lesions are firstly diagnosed even in older patients and can exist over a long time without symptoms. Thus, significant atrial shunt lesions are an important differential diagnosis not only in young patients. Every effort must be made by the echocardiographer to find the underlying disease if an enlarged right heart is detected.

For future studies, corrections for clinical states with high cardiac output would be of interest. This could be done by measuring the VTI of the LVOT and comparing it with the VTI of the RVOT. This parameter would be one step closer to Qp:Qs, but without the error prone measurements of the outflow tracts.

\section{Limitations}

The retrospective design of this study has several limitations. The exact position of the PW-Doppler sample volume could not be evaluated retrospectively. A different position of the sample volume in the pulmonary artery or in the distal RVOT can change the value. Clinical situations with high cardiac output such as pregnancy, fever, hyperthyroidism, anemia, and dialysis shunts are expected to present with high VTIs without presence of an atrial shunt lesion. Due to lack of clinical data and the retrospective design of the study, these patients could not be excluded from the analysis.

The bias of these limitations was reduced by choosing a longer period of time and thus a larger number of patients.

\section{Conclusion}

In this large retrospective analysis, we could show that a low RVOT-VTI predicted the absence, while a high RVOT-VTI predicted the presence of significant atrial shunt lesions. The parameter should be applied in all patients with suspicion for atrial shunt lesions where calculation of Qp:Qs is impossible.

Funding Open access funding provided by Medical University of Vienna.

Conflict of interest V. Dannenberg, G. Goliasch, C. Hengstenberg, T. Binder, H. Gabriel, and M. Schneider declare that they have no competing interests.

Open Access This article is licensed under a Creative Commons Attribution 4.0 International License, which permits use, sharing, adaptation, distribution and reproduction in any medium or format, as long as you give appropriate credit to the original author(s) and the source, provide a link to the Creative Commons licence, and indicate if changes were made. The images or other third party material in this article are included in the article's Creative Commons licence, unless indicated otherwise in a credit line to the material. If material is not included in the article's Creative Commons licence and your intended use is not permitted by statutory regulation or exceeds the permitted use, you will need to obtain permission directly from the copyright holder. To view a copy of this licence, visit http://creativecommons.org/licenses/by/4.0/.

\section{References}

1. Motiwala A, Fatimi SH, Akhtar N, Perveen S, Khan MZ, Atiq M. Patients with congenital atrial septal defects: effect of age at repair and defect size on pulmonary artery pressures prior to repair. Thorac Cardiovasc Surg. 2011;59(5):281-6.

2. Geggel RL. Clinical detection of hemodynamically significant isolated secundum atrial septal defect. J Pediatr. 2017;190:261-264.e1.

3. Engelfriet PM, Duffels MG, Moller T, Boersma E, Tijssen JG, Thaulow E, et al. Pulmonary arterial hypertension in adults born with a heart septal defect: the Euro heart survey on adult congenital heart disease. Heart. 2007;93(6):682-7.

4. EUROCAT. Special report: congenital heart defects in europe 2000-2005. 2009. http://www.eurocat-network.eu/ content/Special-Report-CHD.pdf. Accessed 12 May 2019.

5. GiblettJP, Abdul-Samad O, Shapiro LM, Rana BS, CalvertPA. Patent foramen ovale closure in 2019. Interv Cardiol. 2019;14(1):34-41.

6. Muta H, Akagi T, Egami K, Furui J, Sugahara Y, Ishii M, et al. Incidence and clinical features of asymptomatic atrial septal defect in school children diagnosed by heart disease screening. Circ J. 2003;67(2):112-5.

7. Silvestry FE, Cohen MS, Armsby LB, Burkule NJ, Fleishman CE, Hijazi ZM, et al. Guidelines for the echocardiographic assessment of atrial septal defect and patent foramen ovale: from the American society of echocardiography and society for cardiac angiography and interventions. JAm SocEchocardiogr. 2015;28(8):910-58.

8. Kronzon I, Tunick PA, Freedberg RS, Trehan N, Rosenzweig BP, Schwinger ME. Transesophageal echocardiography is superior to transthoracic echocardiography in the diagnosis of sinus venosus atrial septal defect. J Am Coll Cardiol. 1991;17(2):537-42.

9. Lang RM, Badano LP, Mor-Avi V, Afilalo J, Armstrong A, Ernande L, et al. Recommendations for cardiac chamber quantification by echocardiography in adults: an update from the American society of echocardiography and the European association of cardiovascular imaging. J Am Soc Echocardiogr. 2015;28(1):1-39.e14. 
10. Schneider M, Binder T. Echocardiographic evaluation of the right heart. Wien Klin Wochenschr. 2018;130(13-14):413-20.

11. De Simone R, Iarussi D, Haberbosch W, Scialdone A, Irace L, Iacono A. Clinical usefulness of the Doppler-echocardiographic method for evaluating intracardiac shunts. Combined Doppler and hemodynamic study. Cardiologia. 1989;34(8):689-94.

12. Vargas Barron J, Sahn DJ, Valdes-Cruz LM, Lima CO, Goldberg SJ, Grenadier E, et al. Clinical utility of two-dimensional doppler echocardiographic techniques for estimating pulmonary to systemic blood flow ratios in children with left to right shunting atrial septal defect, ventricular septal defect or patent ductus arteriosus. J Am Coll Cardiol. 1984;3(1):169-78.

13. Kitabatake $A$, Inoue $M$, Asao $M$, Ito $H$, Masuyama $T$, Tanouchi J, et al. Noninvasive evaluation of the ratio of pulmonary to systemic flow in atrial septal defect by duplex Doppler echocardiography. Circulation. 1984;69(1):73-9.

14. Baumgartner H, Bonhoeffer P, De Groot NM, de Haan F, Deanfield JE, Galie N, et al. ESC Guidelines for the management of grown-up congenital heart disease (new version 2010). Eur Heart J.2010;31(23):2915-57.

15. Stout KK, Daniels CJ, Aboulhosn JA, Bozkurt B, Broberg CS, Colman JM, et al. 2018 AHA/ACC guideline for the management of adults with congenital heart disease: executive summary: a report of the American college of cardiology/ American heart association task force on clinical practice guidelines. Circulation. 2019;139(14):e637-e97.

16. Beigbeder JY, Wittenberg O, Messner Pellenc P, Voisin M, Gallay P, Grolleau R, et al. Evaluation of the left-to-right shunt in interatrial septal defects (ostium secundum) using Doppler echocardiography. Apropos of 12 cases. Ann Cardiol Angeiol (Paris). 1989;38(9):549-54.

17. Stern H, Vogel M, Emmrich P, Buhlmeyer K. The accuracy of Doppler echocardiography determination of shunt volume in children with isolated atrial septal defect and left-right shunt. ZKardiol. 1989;78(10):674-9.

18. Rudski LG, Lai WW, Afilalo J, Hua L, Handschumacher MD, Chandrasekaran K, et al. Guidelines for the echocardiographic assessment of therightheartin adults: a reportfrom the American society of echocardiography endorsed by the European association of echocardiography, a registered branch of the European society of cardiology, and the Canadian society of echocardiography. J Am Soc Echocardiogr. 2010;23(7):685-713. quiz 86-8.

19. Lang RM, Badano LP, Mor-Avi V, Afilalo J, Armstrong A, Ernande L, et al. Recommendations for cardiac chamber quantification by echocardiography in adults: an update from the American society of echocardiography and the European association of cardiovascular imaging. Eur Heart J Cardiovasc Imaging. 2015;16(3):233-70.

20. Engelfriet P, Meijboom F, Boersma E, Tijssen J, Mulder B. Repaired and open atrial septal defects type II in adulthood: an epidemiological study of a large European cohort. Int J Cardiol. 2008;126(3):379-85.

Publisher's Note Springer Nature remains neutral with regard to jurisdictional claims in published maps and institutional affiliations. 